\title{
Corrélats neurophysiologiques de l'imagerie motrice
}

\author{
Christian Collet $^{1}$, Franck Di Rienzo ${ }^{1}$, Nady Hoyek $^{1}$ et Aymeric Guillot ${ }^{1,2}$ \\ 1 Centre de recherche et d'innovation sur le sport, EA 647, Performance Motrice, Mentale et du Matériel (P3M), \\ Université Claude Bernard Lyon 1, Université de Lyon, France \\ 2 Institut universitaire de France, 103 boulevard Saint-Michel, 75005, Paris, France
}

Reçu le 24 juin 2012 - Accepté le 6 novembre 2012

\begin{abstract}
Résumé. Dans les protocoles expérimentaux portant sur l'imagerie motrice, la question de l'évaluation effective du travail mental effectué par les participants demeure une question récurrente pour le chercheur. De même, le praticien doit pouvoir disposer de méthodes lui permettant d'évaluer la qualité de l'imagerie motrice, qu'il soit entraîneur et l'utilise à l'amélioration de la performance ou clinicien et en prescrive la pratique pour favoriser le recouvrement des fonctions motrices. Si les évaluations subjectives, faites par les participants, et la passation de questionnaires restent des outils fréquemment utilisés, on dispose aussi d'indicateurs physiologiques centraux et périphériques. La neuroimagerie identifie les corrélats structuraux et fonctionnels de l'imagerie motrice, mais cette méthodologie n'est pas ambulatoire et reste réservée aux protocoles de laboratoire. Les indicateurs périphériques apportent des signes objectifs du travail mental dont il faut traiter les données pour pouvoir les exploiter afin d'évaluer la qualité de l'imagerie. L'objectif de cet article est de présenter les caractéristiques et limites de ces familles méthodologiques.
\end{abstract}

Mots clés : Imagerie motrice, imagerie par résonance magnétique fonctionnelle, activité neurovégétative, mouvements oculaires, performance motrice

\begin{abstract}
Neurophysiological correlates of motor imagery.
Measuring individual differences in motor imagery ability as well as imagery vividness remains a recurring question that must be resolved by experimenters, coaches, and athletes. Practically, such evaluation is required to control how using imagery efficiently to improve motor performance and/or enhance motor recovery. While the use of self-report scales and imagery questionnaires remains useful tools, a wide range of physiological recordings from the central and autonomic nervous systems correlate motor imagery and can also be considered to evaluate motor imagery quality. Neuroimaging methods provide objective information about structural and functional correlates of motor imagery. However, these are not ambulatory and thus remain confined to laboratory paradigms. Information from peripheral nervous system, either somatic or vegetative, is of critical interest since it is directly related to mental activity and can be assessed to evaluate motor imagery quality. This paper aimed at examining the major features and limits of these methods.
\end{abstract}

Key words: Motor imagery, functional magnetic resonance imaging, autonomic nervous system activity, eye movements, motor performance

\section{Introduction}

L'imagerie motrice est la représentation mentale d'une action sans production concomitante de mouvement. Fréquemment utilisée dans l'entraînement sportif comme dans celui de la rééducation motrice, ses domaines d'application sont variés et ont fait l'objet de plusieurs modèles théoriques et/ou synthétiques (e.g., Martin, Moritz, \& Hall, 1999; Munroe, Giaccobi, Hall, \& Weinberg, 2000; Holmes \& Collins, 2001; Guillot \&
Collet, 2008). Son efficacité dépend en partie des capacités d'imagerie, différentes d'un sujet à l'autre. Certains parviennent facilement à se représenter les séquences d'un mouvement alors que d'autres éprouvent des difficultés à concevoir leur propre image ou celle d'un d'autre en train de l'exécuter. Une des questions récurrentes est d'arriver à évaluer les capacités d'imagerie à l'aide de méthodes objectives. Il existe un ensemble de tests dont le but est d'y parvenir. Tous apportent des informations complémentaires mais souvent à partir d'une 
auto-évaluation. L'enjeu serait de proposer d'autres outils qui pourraient les compléter. Dans les protocoles scientifiques, la qualité du travail mental est une variable à contrôler pour s'assurer que ses effets éventuels sur les variables dépendantes puissent être attestés et validés. De même, dans la pratique sportive, si on suppose que l'imagerie motrice influence directement la performance réelle, elle doit être contrôlée.

Évaluer la qualité d'un processus cognitif reste une entreprise complexe. Nous décrirons plusieurs outils complémentaires, en précisant leurs attributions et leurs limites. Auparavant, nous devons décrire les facteurs qui définissent une représentation mentale de qualité.

\section{Caractéristiques intrinsèques des représentations mentales}

La qualité d'une représentation mentale se base sur trois propriétés : sa vivacité, son exactitude et son contrôle (Moran, 1993; Morris, Spittle, \& Watt, 2005). Ce sont les dimensions de l'imagerie. La vivacité représente sa netteté et sa précision. L'exactitude retranscrit sa fidélité par rapport au modèle. Le contrôle atteste la capacité à manipuler et à transformer l'image. Chaque dimension est indépendante l'une de l'autre : la représentation peut être nette (vivacité) sans être fidèle à la réalité de la scène (exactitude). La qualité de l'imagerie implique une évaluation simultanée des trois dimensions. La conservation de l'organisation temporelle du mouvement est aussi un déterminant de la qualité de l'imagerie (pour revues, voir Guillot \& Collet, 2005a; Guillot, Hoyek, Louis, $\&$ Collet, 2012). Sans consigne particulière, il y a isochronie entre le mouvement imaginé et son exécution réelle. On le vérifie facilement par chronométrie mentale, méthode fiable et reproductible (e.g., Malouin, Richards, Durand, \& Doyon, 2008). Il suffit de comparer la durée d'exécution d'un mouvement à celle de sa simulation mentale, si possible en incluant des mesures intermédiaires, correspondant à ses séquences constitutives. Même si plusieurs facteurs influencent cette congruence temporelle, modifier la vitesse du mouvement imaginé altère la vitesse du mouvement réel, soit en l'augmentant, soit en la diminuant (Boschker, Bakker, \& Rietberg, 2000; Louis, Guillot, Maton, Doyon, \& Collet, 2008). Évaluer les autres dimensions de l'imagerie est plus complexe. Les questionnaires psychologiques, les entretiens ou encore les auto-évaluations sont des moyens faciles d'utilisation et généralement validés scientifiquement. Ces méthodes restent toutefois subjectives et les participants peuvent avoir des difficultés pour retranscrire précisément les caractéristiques de leurs représentations mentales. Les combiner avec une approche plus objective est donc un enjeu majeur.

Les corrélats physiologiques de l'imagerie motrice apportent davantage d'objectivité dans l'évaluation de sa qualité. L'activité du système nerveux central, électromagnétique, métabolique ou électrique nous informe sur les structures impliquées et permet d'identifier des réseaux différents selon la qualité et le type d'imagerie (Solodkin, Hlustik, Chen, \& Small, 2004; Guillot, Collet, Nguyen, Malouin, Richards, \& Doyon, 2008). Une approche plus fonctionnelle consiste à enregistrer des indicateurs périphériques avec les corrélats musculaires, oculaires et végétatifs de l'imagerie motrice (Fig. 1). Chaque méthode offre des procédures complémentaires d'évaluation, leur combinaison augmentant considérablement sa fiabilité (Guillot \& Collet 2005b).

\section{Recueil de l'activité du système nerveux central}

L'imagerie par résonance magnétique fonctionnelle (IRMf) fournit des images tridimensionnelles de la distribution de l'activité métabolique cérébrale instantanée, dont la résolution spatiale atteint maintenant le millimètre cube. L'électroencéphalographie et la magnétoencéphalographie enregistrent l'activité électrique et magnétique du cerveau, avec une résolution temporelle élevée ${ }^{1}$, de l'ordre de la milliseconde. Comparer les activations cérébrales des mouvements réels et imaginés permet de mieux comprendre les processus de formation des représentations mentales (pour revues, voir Lotze \& Halsband, 2006; Hanakawa Dimyan, \& Hallett, 2008; Munzert, Lorey, \& Zengraf, 2009; Guillot, Di Rienzo, \& Collet, 2012) (Fig. 2). La correspondance des activations cérébrales durant l'exécution réelle et l'imagerie motrice permet d'évaluer la qualité de la représentation mentale. Ces données apportent des arguments en faveur de leur équivalence fonctionnelle (Jeannerod, 1994; Grèzes \& Decety, 2001). Le facteur limitant de ces méthodes est qu'elles restent confinées aux mouvements segmentaires (séquence d'opposition des doigts, par exemple).

\section{1 Équivalence fonctionnelle entre mouvements réels et simulés}

Une des premières publications dédiées à l'imagerie motrice utilisant l'IRMf date de 1980. Roland, Larsen, Lassen et Skinhoj (1980) ont alors révélé l'activation de l'aire prémotrice et de l'aire motrice supplémentaire. D'autres études ont ensuite confirmé ce résultat (Decety

1 La stimulation magnétique transcrânienne (SMT) est une technique non invasive qui consiste à appliquer une impulsion magnétique sur une structure cérébrale à l'aide d'une bobine. On enregistre, puis on analyse les potentiels moteurs évoqués dans les muscles concernés. Ici, nous n'étudions que les signaux cérébraux intrinsèques, ceux que le système nerveux génère. Un article de ce numéro thématique porte sur la SMT et son utilisation dans l'étude de l'imagerie motrice. 


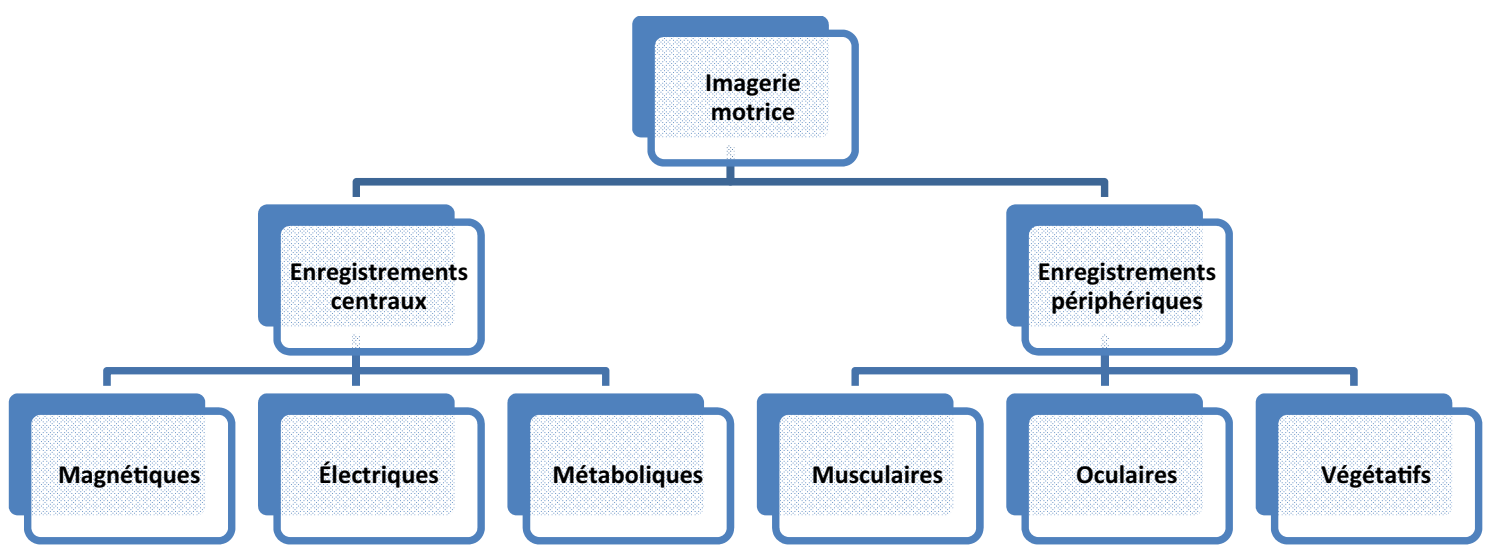

Fig. 1. Résumé des corrélats physiologiques de l'imagerie motrice. Les indicateurs centraux apportent des informations structurales, en décrivant les réseaux de neurones qui contrôlent l'imagerie motrice. Les signes périphériques permettent une évaluation fonctionnelle grâce aux activités musculaire, oculaire et végétative enregistrées simultanément à la représentation mentale d'un mouvement.

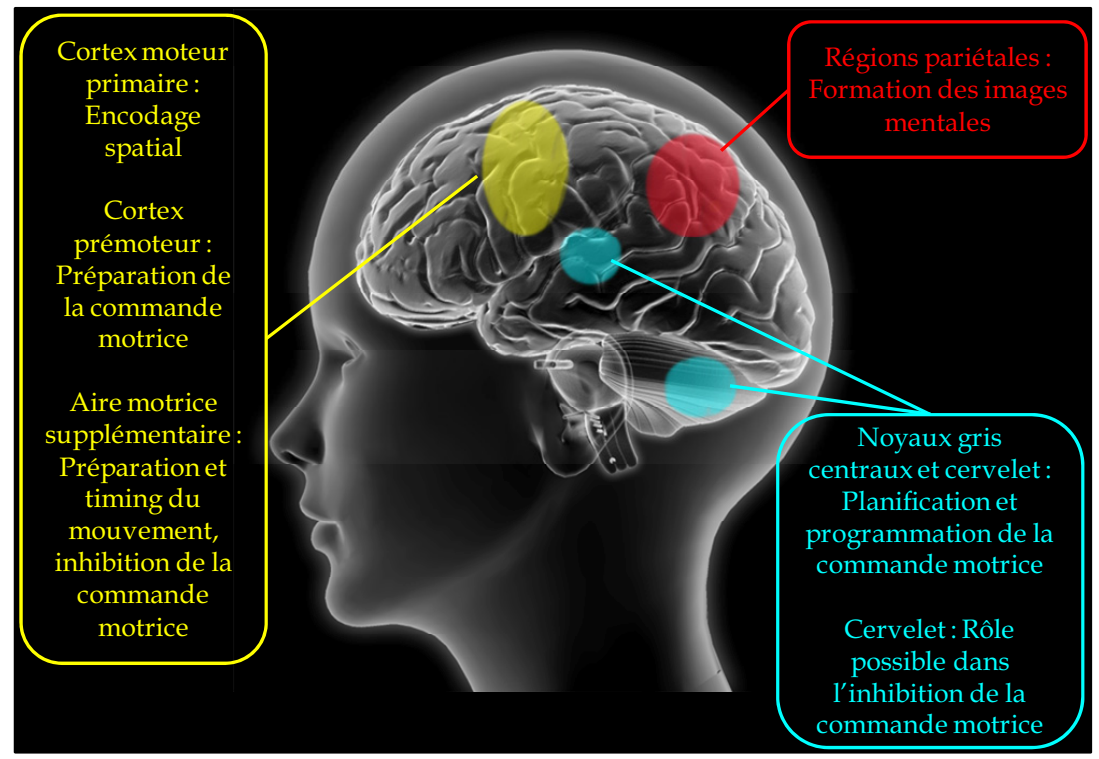

Fig. 2. Activations cérébrales observées lors de l'imagerie motrice (adapté de Guillot, et al., 2012).

et al., 1994; Gerardin et al., 2000; Guillot et al., 2008; Hanakawa, Dimyan, \& Hallett, 2003; Lotze et al., 1999; Munzert et al., 2009; Solodkin et al., 2004). L'implication de l'aire motrice primaire a suscité davantage de controverses (pour revues, voir Lotze \& Halsband, 2006; Sharma, Jones, Carpenter, \& Baron, 2008; Munzert et al., 2009; Lotze \& Zentgraf, 2010). Certains n'ont pas enregistré de changement d'activité (Binkofski et al., 2000; Gerardin et al., 2000; KuhtzBushbeck, Mahnkopf, Holzknecht, Siebner, Ulmer, \& Jansen, 2003; Hanakawa et al., 2003, 2008), alors que d'autres ont observé son activation (Leonardo et al., 1995; Porro, Cettolo, Francescato, \& Baraldi, 2000; Lotze et al., 1999; Solodkin et al. 2004; Guillot et al., 2009). Selon Ehrsson, Geyer et Naito (2003), imaginer des mouvements des doigts, des pieds et de la langue, active spécifiquement les régions du cortex moteur primaire qui les contrôlent. Ceci serait potentiellement lié à son organisation somatotopique, les aires les plus larges étant les plus susceptibles d'être activées. D'autres facteurs pourraient moduler son implication, comme les instructions données aux participants, le niveau d'expertise, la capacité d'imagerie individuelle, ou encore la complexité des mouvements imaginés (Kuhtz-Buschbeck et al., 2003; Sharma, Pomeroy, \& Baron, 2006 ; Lotze \& Zentgraf, 2010). Une consigne précise, allant jusqu'au paramétrage de l'action, un niveau de pratique élevé, une forte capacité d'imagerie et un mouvement facile constituent des conditions susceptibles d'activer l'aire motrice primaire. Inversement, une consigne globale, un niveau de pratique novice, une faible capacité d'imagerie et un mouvement complexe concourent plutôt à sa 
non-activation. Cette hypothèse pourrait être facilement testée expérimentalement.

L'équivalence fonctionnelle entre mouvement réels et imaginés s'observe également dans d'autres régions corticales et sous-corticales, en particulier le cervelet et les noyaux gris centraux (Gerardin et al., 2000; Munzert et al., 2009), les régions pariétales associatives inférieures et supérieures (Hanakawa, Immisch, Toma, Dimyan, Van Gelderen, \& Hallett, 2003; Gao, Duan, \& Chen, 2011). La pathologie confirme le rôle des structures pariétales dans la simulation mentale d'une action, leur lésion altérant la capacité à se représenter l'action (Sirigu, Duhamel, Cohen, Pillon, Dubois, \& Agid, 1996; Malouin, Belleville, Richards, Desrosiers, \& Doyon, 2004; Sabate, Gonzalez, \& Rodriguez, 2007). Schwoebel, Boronat et Coslett (2002) ont décrit le cas d'un patient atteint d'une lésion pariétale bilatérale, qui effectuait inconsciemment le mouvement qu'on lui demandait d'imaginer. La commande motrice serait donc construite pendant l'imagerie puis inhibée pour ne pas la transmettre dans les voies efférentes (pour revue, voir Guillot, Di Rienzo, Moran, MacIntyre, \& Collet, 2012). Le cortex pariétal aurait une fonction importante dans ce processus.

La neuroimagerie montre que les réseaux neuronaux contrôlant l'exécution réelle d'un mouvement et sa simulation mentale sont en partie communs. Macuga et Frey (2012) ont observé que les configurations d'activations lors de l'imagerie motrice correspondaient à une partie de celles contrôlant l'exécution du même comportement. Celles liées à son observation ressemblaient aussi à une partie des activations induites par l'imagerie motrice. Les réseaux nerveux impliqués dans ces trois tâches confirment l'équivalence fonctionnelle entre exécuter, se représenter et observer.

\subsection{Configurations d'activations différenciées}

Malgré les similitudes structurales et fonctionnelles, exécution réelle et simulée mentalement ne sollicitent pas exactement les mêmes réseaux (Hanakawa et al., 2008). Plusieurs études ont comparé les configurations d'activations cérébrales pendant l'imagerie visuelle et kinesthésique (e.g., Solodkin et al., 2004; Binkofski et al., 2000). L'imagerie visuelle active davantage les régions occipitales et pariétales alors que l'imagerie kinesthésique mobilise plutôt les régions motrices corticales, sous-corticales et le cortex pariétal inférieur (Guillot et al., 2009). Ruby et Decety (2001) ont montré que les régions contrôlant l'imagerie à la première personne (représentation de l'action comme acteur) étaient partiellement différentes de celles activées pendant l'imagerie à la troisième personne (représentation en tant que spectateur $^{2}$ ). Jackson, Meltzoff et Decety (2006) puis Lorey,

${ }^{2} \mathrm{Au}$ cours de cette expérience, dans la condition d'imagerie à la troisième personne, il était demandé aux participants d'imaginer l'expérimentateur faire le mouvement.
Bischoff, Pilgramm, Stark, Munzert, et Zentgraf (2009) ont ensuite mis en évidence que l'activation cérébrale induite par l'imagerie à la première personne se rapprochait davantage de celle observée lors de l'exécution réelle, notamment en raison de l'activation des systèmes moteurs.

Une activation cérébrale partiellement différente a été rapportée lors de la comparaison de sujets experts et débutants : les violonistes novices présentaient un réseau d'activation plus étendu que celui des experts (Lotze, Scheler, Tan, Braun, \& Birbaumer, 2003). Cette différence a également été observée pour une séquence d'opposition des doigts (Milton, Solodkin, Hlustîk, \& Small, 2007; Milton, Small, \& Solodkin, 2008; Ross, Tkach, Ruggieri, Lieber, \& Lapresto, 2003). Enfin, les configurations d'activation sont différentes chez les bons et mauvais imageurs (Guillot et al., 2008). En référence au modèle théorique de l'apprentissage moteur de Doyon et Benali (2005), les mauvais imageurs activeraient conjointement les circuits cortico-striatal et cortico-cérébelleux lors de la simulation mentale, alors que les bons imageurs n'activeraient que le premier.

Le fait qu'imaginer un mouvement et l'exécuter réellement active des zones cérébrales communes permet de comprendre pourquoi la simulation mentale peut faciliter l'apprentissage et améliorer la performance. La neuroimagerie permet d'étudier l'évolution des activations au cours du temps, par exemple lorsque deux enregistrements sont séparés d'une période d'entraînement mental. Les méthodes de neuroimagerie cérébrale rendent alors compte de la plasticité cérébrale induite par le travail mental (e.g., Lotze et al., 2003; Lafleur, Jackson, Malouin, Richards, Evans, \& Doyon, 2002). Jackson, Lafleur, Malouin, Richards et Doyon (2003) ont observé une augmentation de l'activité corticale orbitofrontale et une diminution des activations cérébelleuses après un entraînement intensif par imagerie motrice. Les bons imageurs ont présenté une activation plus marquée du cortex pariétal et des régions prémotrices ventrolatérales, secteurs bien identifiés pour leur rôle dans la génération des images mentales (Guillot et al., 2008). La répétition mentale aurait donc bien la capacité à induire la plasticité cérébrale.

$\mathrm{Au}$ regard des études mentionnées dans cette partie, notre compréhension des processus cérébraux impliqués dans la simulation mentale du mouvement est facilitée par les études de neuroimagerie. Elles ont permis de valider objectivement un certain nombre de questions, comme l'identité structurale entre mouvement et représentation du mouvement, l'identification de réseaux spécifiques en fonction du type d'imagerie ou de la capacité individuelle d'imagerie. Ces méthodes restent orientées vers la recherche fondamentale. Leur caractère peu ambulatoire et leur lien réduit avec la motricité corporelle globale, notamment la motricité sportive, les rendent peu opérationnelles dans ce domaine, bien qu'on puisse généraliser les résultats des protocoles de laboratoire à des situations plus globales comme les techniques sportives. Mais, pour le 
confirmer, une alternative consiste à enregistrer d'autres indicateurs, en particulier ceux qui révèlent les caractéristiques de la commande centrale à travers ses manifestations périphériques. L'électromyographie renseigne sur la nature de la commande nerveuse adressée aux muscles, l'oculographie sur les mouvements oculaires associés à l'imagerie motrice, et l'activité neurovégétative périphérique sur les commandes destinées aux effecteurs végétatifs précédant et accompagnant le mouvement.

\section{Recueil de l'activité du système nerveux périphérique}

\subsection{Corrélats électromyographiques de l'imagerie motrice}

Existe t-il une activité musculaire corrélative de l'imagerie motrice qui serait attestée par l'électromyographie (EMG)? Compte tenu que l'imagerie ne débouche pas sur l'exécution du mouvement, elle ne devrait pas générer une activité musculaire différente de la condition contrôle. Des études le confirment (e.g., Decety, Jeannerod, Durozard, \& Baverel, 1993; Ranganathan, Siemionow, Liu, Sahgal, \& Yue, 2004; Mulder, de Vries, \& Zijlstra, 2005 ; Personnier, Paizis, Ballay, \& Papaxanthis, 2007 ) et d'autres s'appuient sur cet argument pour justifier que les activations cérébrales observées ne sont pas dues à une quelconque activité musculaire associée, tonique ou phasique, mais bien à la formation des images mentales. Cette vérification est parfois faite en amont, pour s'assurer que les participants sont capables d'imaginer un mouvement sans accompagnement musculaire, en particulier dans les muscles sollicités par le mouvement imaginé (Hanakawa et al., 2003; Jackson et al., 2003). D'autres études couplent l'activité électromyographique aux enregistrements centraux où l'EMG a aussi une fonction de contrôle (Kleber, Birbaumer, Veit, Trevorrow, \& Lotze, 2007; Lotze et al., 2003). Plusieurs autres travaux ont considéré l'EMG comme une variable dépendante afin de tester l'hypothèse préalablement posée par Jeannerod (1994) : identifier une activité musculaire résiduelle, résultant d'une inhibition incomplète de la commande motrice. Lors de la simulation mentale, Gandevia Wilson, Inglis et Burke (1997), Jacobson (1931) et Jowdy et Harris (1990) ont enregistré une activité musculaire subliminale (pour revue, voir Guillot, Lebon, \& Collet, 2010) qui intègre les caractéristiques de la commande musculaire du mouvement. Selon Harris et Robinson (1986), l'imagerie à la première personne a généré une activité musculaire plus intense que la représentation à la troisième personne. En imagerie interne, l'intensité de l'activité musculaire subliminale était proportionnelle à l'effort imaginé : plus la charge à mobiliser mentalement était lourde, plus l'activité musculaire était élevée, tout en demeurant subliminale, c'est-à-dire incapable de générer une contraction réelle (Bakker, Boschker, \& Chung, 1996 ; Boschker, 2001; Slade, Landers, \& Martin, 2002). On aurait pu conclure à une simple élévation du tonus musculaire dans les muscles concernés par le mouvement imaginé, comme ce peut être le cas lorsqu'on observe quelqu'un en train d'exécuter un mouvement (imprégnation perceptivo-motrice). Cependant, l'activité EMG enregistrée pendant l'imagerie différencie les régimes de contraction, suivant qu'on se représente une contraction concentrique, isométrique ou excentrique (Guillot, Lebon, Rouffet, Champely, Doyon, \& Collet, 2007 ; Lebon, Rouffet, Collet, \& Guillot, 2008). L'argument qui valide ce résultat est que les différences observées mentalement reproduisent celles enregistrées lors des mouvements réels. Enfin, une activité musculaire subliminale différente du niveau basal a aussi été enregistrée dans les muscles, synergistes et fixateurs.

Ces résultats divergents s'expliqueraient par des facteurs méthodologiques liés à l'EMG, mais aussi par les consignes le type d'imagerie, ou encore l'intensité des mouvements imaginés qui pourraient induire une activité musculaire subliminale. Dickstein, Gazit-Grunwald, Plax, Dunsky et Marcovitz (2005) et Li Kamper, Stevens et Rymer (2004) ne l'ont enregistrée que chez certains participants. La simulation mentale d'un mouvement s'accompagnerait d'une activité musculaire de faible intensité dans les muscles concernés, lorsque l'imagerie est kinesthésique. Si les étapes de préparation de la commande motrice sont similaires lors de l'imagerie et de la pratique réelle, aucune étude n'a cependant reproduit la configuration triphasique de la contraction et du relâchement des muscles agonistes et antagonistes, respectivement (Murphy, Nordin, \& Cumming, 2008). Enfin, l'activité EMG recueillie ne semble pas corrélée aux capacités d'imagerie (Guillot et al., 2007). Par conséquent, il est difficile de la considérer comme un témoin de la qualité du travail mental.

\subsection{Mouvements oculaires}

Deux études ont montré que la simulation mentale s'accompagne de mouvements oculaires comparables à ceux recueillis durant la perception (e.g., Laeng \& Teodorescu, 2002; Rodionov, Zislin, \& Elidan, 2004). Cette thématique a ensuite été quel que peu abandonnée, avant d'être récemment reconsidérée par plusieurs équipes de recherche. Que les yeux soient ouverts ou fermés, Heremans, Helsen et Feys (2008) ont alors montré la similitude des mouvements oculaires pendant la simulation mentale d'un mouvement et son exécution réelle. Dans ces deux conditions, par exemple, la vision anticipe la position de la main pour faciliter la saisie d'un objet. Cependant, ces résultats n'ont pas été systématiquement confirmés (e.g., Debarnot, Valenza, Champely, Scilingo, De Rossi, \& Guillot, 2011; Gueugneau, Crognier, \& Papaxanthis, 2008), même si plusieurs études valident l'hypothèse de l'identité des mouvements oculaires lors de l'exécution réelle et de sa représentation mentale (Heremans et al., 2011, 2012). Pour généraliser la validation de cette hypothèse, Mast et Kosslyn (2002) proposent d'étudier 
comment la motricité oculaire s'organise lorsque le stimulus perçu est imaginé dans une orientation différente de celle qui a permis son encodage. Cet indicateur pourrait constituer un moyen objectif d'évaluation de la qualité du travail mental. Il pourrait potentiellement améliorer l'efficacité de l'imagerie, en étant utilisé comme information rétroactive.

\subsection{Corrélats neurovégétatifs au cours de l'imagerie motrice}

Le système neurovégétatif régule les fonctions vitales de l'organisme. Partant de cette définition, ses rapports avec l'imagerie motrice ne s'imposent pas de façon évidente. Nous allons donc décrire comment les relations entre physiologie neurovégétative et imagerie motrice peuvent être établies puis analysées afin de fournir des corrélats de l'activité mentale. Cela nous obligera à décrire les avancées récentes de la physiologie neurovégétative, en remettant en cause, par exemple, la notion de tonus sympathique général. C'est un passage obligé avant d'établir la relation entre imagerie motrice et indicateurs végétatifs.

Le système nerveux végétatif (SNV) est composé de voies viscérales afférentes dont les centres d'intégration sont dans le tronc cérébral, l'hypothalamus et le cortex. Son organisation comprend également des voies efférentes subdivisées en systèmes orthosympathique, parasympathique et entérique qui régulent les fonctions viscérales par des signaux nerveux et des neurotransmetteurs spécifiques (McCorry, 2007). Les effecteurs sont les organes internes, comme le cœur, mais aussi la musculature lisse, les glandes exo- et endocrines. L'innervation de la peau fait exception au principe de la double innervation. C'est un constat important sur lequel nous reviendrons.

La branche orthosympathique mobilise les ressources de l'organisme pour pallier toute demande énergétique nécessaire (fonction catabolique). La branche parasympathique a une fonction antagoniste qui maintient et restaure les ressources de l'organisme à un niveau compatible avec les fonctions vitales (fonction anabolique). Pour Wallin et Fagius (1986), «le système nerveux végétatif est hautement différencié, rapidement activé, avec des subdivisions spécialisées dans la régulation des fonctions organiques, en réponse aux demandes changeantes du milieu intérieur et des stimulations externes $»$. La balance ortho/parasympathique reste une donnée habituelle de la physiologie végétative mais doit être nuancée car des coactivations sont possibles (Backs, 1998), de même qu'une modification d'activité dans une branche peut laisser l'autre inchangée. Pour Bernston, Cacioppo et Quigley (1991), les relations entre activités sympathique et parasympathique sont ainsi plus complexes qu'une simple réciprocité.

La description classique de la physiologie végétative suscite deux critiques majeures. Au niveau anatomique, la subdivision proposée par Xavier Bichat (1802) sépare artificiellement le système nerveux somatique qui contrôle la vie de relation, du végétatif qui règle la vie viscérale. Par conséquent, le végétatif serait implicitement indépendant de la volonté et de la conscience. Néanmoins, il reste indispensable au fonctionnement adéquat de la vie de relation puisqu'elle repose sur sa fonction métabolique. En contrôlant l'activation générale de l'organisme, le système végétatif conditionne l'efficacité comportementale. Un comportement n'atteint pas son but si l'activation n'est pas suffisante. Inversement, une suractivation orthosympathique entraîne une baisse de performance, comme si du bruit était introduit dans le système, ce qui a conduit à faire du stress la conséquence d'une sur-sollicitation orthosympathique. Nous établissons là une première relation entre le SNV et un état mental particulier.

Les avancées de la physiologie végétative (Morisson, 2001; Vallbo Hagbarth, \& Wallin, 2004) ont montré que «les premières conceptions du système nerveux sympathique en tant qu'effecteur monolithique, activé globalement dans des situations exigeant une réponse rapide à toute situation d'agression, ont été éclipsées par un modèle d'organisation disposant d'une vaste gamme de canaux de sortie fonctionnellement spécifiques, pouvant être simultanément activés ou inhibés dans des combinaisons qui résultent en une situation favorisant le comportement $»$. Porges (1995a) a abouti à la même conclusion en montrant que la branche parasympathique agissait sélectivement sur le système cardiaque selon que l'origine de la commande était le noyau dorsal moteur ou le noyau ambigu. Le premier exerce une fonction modératrice via le nerf vague. L'activité du noyau ambigu est corrélée à des processus d'attention et de réactivité émotionnelle. Il y a donc un lien clair entre les fonctions mentales et l'activité du SNV (Porges, 1995b). Selon Hugdahl (1996), «l'activité végétative qui accompagne l'attention, l'orientation et l'apprentissage a démontré que le système nerveux autonome ne représente pas simplement une partie «non-cognitive » et automatique de la fonction cérébrale ». Même si les réponses sont soumises à l'habituation (Bradley, 2009), un stimulus connu est rapidement identifié et induit une réponse végétative parce qu'elle a une signification cognitive et émotionnelle. Un stimulus nouveau va provoquer une alerte plus intense et, par conséquent, une réponse de plus forte amplitude. Enfin, toute information non perçue ne génère pas de réponse végétative. Le SNV est donc sensible à la signification cognitive et émotionnelle de l'information à laquelle le sujet est confronté.

Après avoir établi les liens entre processus mentaux et activité végétative, nous pouvons considérer que toute information peut être mentalement évoquée, c'està-dire rappelée en l'absence de tout stimulus effectif. Par conséquent, des réponses végétatives identiques à celles induites par une stimulation externe pourraient être obtenues par évocation mentale, à partir d'un traitement dirigé par concepts (ou «top-down » selon les auteurs anglais). L'usage d'une «amorce» selon les psychologues (le «pre-cueing $»$ des neurophysiologistes) devrait aussi permettre de rappeler l'information complète mémorisée, 
grâce à la fonction de reconnaissance. C'est cette fonction qui est attendue de l'observation. Dès lors, nous posons l'hypothèse que la stimulation réelle, sa reconnaissance par observation, ou son évocation mentale, sont susceptibles de générer une activité neurovégétative comparable. Paccalin et Jeannerod (2000) ont observé une augmentation de la fréquence respiratoire chez des personnes qui regardaient des sportifs soulevant un poids à charge croissante ou courant sur un tapis roulant. La fréquence était d'autant plus élevée que la charge était lourde et la course rapide. La variation de la respiration de l'observateur était proportionnelle à celle de l'effort consenti par l'acteur. Bolliet, Collet et Dittmar (2005a) ont montré que l'action, sa représentation mentale et son observation généraient des réponses végétatives comparables, néanmoins avec une plus faible amplitude lors de l'observation et de l'imagerie motrice, sans doute parce que ces opérations mentales ne sont pas suivies de l'action. Que ce soit pour l'observation ou la représentation mentale, ces processus sont basés sur une relation entre le mouvement observé et son modèle interne chez l'observateur, modèle qui pourrait également lui permettre de simuler mentalement l'action (Iacoboni, Woods, Brass, Bekkering, Mazziotta, \& Rizzolatti, 1999). L'action simulée peut donc induire une activité perceptive identique à celle ordinairement générée par son exécution (Hesslow, 2002). Le rappel des informations mémorisées est à la base de l'imagerie motrice. Nous en déduisons que la représentation mentale d'un mouvement devrait générer une activité centrale et végétative comparable à celle enregistrée lors de son exécution effective (Conklin, Tiffany, \& Vrana, 2000; Meister, Krings, Foltys, Müller, Töpper, \& Thron, 2004).

Planification et programmation sont des opérations de préparation de la commande motrice. Leur image motrice provient d'un modèle interne du mouvement généré à partir de la mémoire procédurale. Sont mémorisées les opérations de planification, autrement dit la structure générale du mouvement (le schéma de réponse motrice selon Schmidt, 1975) et de programmation (la spécification des paramètres d'exécution, comme l'amplitude, la direction ou la force), même si elle nécessite d'être ajustée au contexte ${ }^{3}$ (Paillard, 1982). Simultanément, le système végétatif règle son paramétrage énergétique en mobilisant les ressources de l'organisme par anticipation. Dès les opérations mentales de préparation du geste, nous enregistrons des variations végétatives. Nous en avons de multiples preuves expérimentales.

\footnotetext{
3 Dans la pratique sportive, la programmation peut être mémorisée si l'habileté est plutôt fermée. Franchir une barre en hauteur implique de paramétrer la technique de saut pour une hauteur donnée et la spécification des paramètres d'exécution peut être mémorisée. Pour une habileté ouverte, l'incertitude liée au milieu ou à la présence d'adversaires et de partenaires exige de pouvoir ajuster le paramétrage en temps réel, même s'il peut être globalement prévu en fonction de la configuration de la situation.
}

Tremayne et Barry (2001) qui ont enregistré l'activité électrodermale et cardiaque chez des tireurs au pistolet, ont montré qu'elle variait en fonction de l'activation nécessaire pour tirer : une augmentation de la fréquence cardiaque et de la conductance cutanée atteste la mobilisation énergétique, relativement faible pour cette activité. En revanche, chez des haltérophiles, cette mobilisation représente une augmentation de la fréquence cardiaque dépassant de $30 \%$ la fréquence de référence (Collet, Guillot, Bolliet, \& Dittmar, 2006). Enfin, la décélération cardiaque précédant le tir est également observée et corrélée aux processus attentionnels (Porges, 1995a; Jennings \& Van der Molen, 2005; Bradley, 2009). Ces deux exemples montrent que le SNV répond à la représentation mentale du geste.

Méthodologiquement, nous quantifions l'activité végétative de deux manières :

- par les variations du niveau de base qui représentent la mobilisation énergétique (variation tonique);

- par les réponses que l'activité mentale induit, comme la focalisation de l'attention nécessaire à la construction de l'image motrice (variation phasique).

Cette méthode a permis de montrer que les haltérophiles séparent la préparation de leur mouvement en deux phases. D'abord, on enregistre une bradycardie marquée, attestant une élévation de l'attention, suivie d'un retour au niveau de base puis une augmentation progressive de la fréquence cardiaque, témoin de la mobilisation énergétique nécessaire à l'exécution à venir. La commande motrice est élaborée par les centres somatiques du SNC simultanément à la mobilisation énergétique nécessaire à son exécution effective, contrôlée par les centres végétatifs. Le SNC co-programme donc le mouvement via les efférences somatiques et végétatives (Mogenson, Jones, \& Yim, 1980). Le paramétrage somatique du mouvement est effectué par le réseau intégrant les structures associatives frontales, pariétales et le cervelet latéral (Paillard, 1982) alors que le paramétrage végétatif est réglé par les cortex orbito-frontal, cingulaire antérieur et insulaire. Leurs commandes atteignent les effecteurs végétatifs par des voies efférentes relayant dans le tronc cérébral et la moelle épinière, avant d'atteindre les organes par deux cellules faisant synapse dans un ganglion.

La représentation du but à atteindre est sans doute la première étape de préparation du mouvement, lors de laquelle sont mobilisées les ressources énergétiques et mentales exigées par l'action. Le système orthosympathique serait le témoin de la mobilisation énergétique. La branche parasympathique serait activée en parallèle, à travers le sous-système du noyau ambigu qui contrôlerait la mobilisation de l'attention (Porges, 1995b). En tir (arc, pistolet), Deschaumes-Molinaro, Dittmar et Vernet-Maury (1992) ont montré l'identité de l'activité végétative enregistrée durant la concentration et l'exécution. Puisque, pour se concentrer, le tireur rappelle mentalement les actions à venir à partir des 
routines mémorisées, c'est bien l'image motrice de l'action qui génère des réponses végétatives comparables à celles enregistrées pendant l'action elle-même (placement des appuis, fixation du bassin, élévation et stabilisation du bras porteur de l'arme et appui sur la gâchette). Dans cette expérience, l'activité du SNV s'est révélée comme un indice de la qualité de l'imagerie motrice. Plus le tir était précis, plus les réponses végétatives enregistrées durant la concentration et l'exécution étaient similaires (Deschaumes-Molinaro, Dittmar, \& Vernet-Maury, 1991).

Des observations comparables ont été effectuées lors de la représentation mentale de la marche sur tapis roulant, avec une augmentation de la fréquence respiratoire et cardiaque (Wuyam, Moosavi, Decety, Adams, Lansing, \& Guz, 1995). Beyer, Weiss, Hansen, Wolf et Seidel (1990) avaient fait le même constat lors de l'imagerie motrice d'un $100 \mathrm{~m}$ en natation. Si l'activité végétative présente une configuration identique lors de l'exécution réelle et imaginée, l'amplitude des variations reste néanmoins plus faible lors de la représentation mentale (Decety et al., 1993; Bolliet et al. 2005b). Récemment quelques contributions ont remis ces résultats en cause. Mulder et al. (2005) ont enregistré l'activité EMG, cardiaque et respiratoire pendant que les sujets s'imaginaient effectuer des flexions de jambe avec une charge de $12,5 \mathrm{~kg}$ dans chaque main. À l'exception de la fréquence respiratoire, aucun autre indice ne s'est révélé différent de la situation contrôle pendant l'imagerie. Demougeot Normand, Denise et Papaxanthis (2009) ont choisi l'activité cardiaque et la pression artérielle comme variables dépendantes pendant l'imagerie de mouvements du tronc des jambes et du poignet. Reconnaissant que la représentation mentale de mouvements cycliques intenses génère une activité végétative, leur objectif était de tester des mouvements discrets et peu intenses. La représentation mentale des mouvements du tronc et des jambes a fait augmenter l'activité cardiaque et la pression artérielle, mais aucune variation n'a été enregistrée durant l'imagerie motrice des mouvements du poignet. De plus, puisque les réponses végétatives révélées pendant l'imagerie des mouvements du tronc et des jambes étaient comparables, ils ont conclu à une réponse végétative forfaitaire. Ces deux études montrent-elles les limites de cette méthode?

Le système orthosympathique a une fonction d'alerte. Par conséquent toute stimulation doit être suffisamment intense pour induire une réponse végétative. Par ailleurs, il est extrêmement sensible à la nouveauté et ses réponses s'éteignent spontanément par habituation. Intensité et nouveauté sont des critères déterminants pour l'obtention de réponses du SNV. Il est vraisemblable que le mouvement du poignet retenu par Demougeot et al. (2009) n'était pas suffisamment intense pour générer une activité végétative détectable. Enfin, on peut aussi poser la question de la finalité puisque les mouvements étudiés n'avaient de but qu'en eux-mêmes. Sans poursuivre un objectif précis, sans finalité réelle, la représentation mentale d'un mouvement ne génère pas facilement de réponses végétatives. Nous les observons pour des gestes orientés vers un résultat à obtenir, ne serait-ce qu'en termes de réussite ou d'échec. L'activité végétative reste fortement corrélée à la signification affective de l'action.

L'expérience de Mulder et al. (2005) était différente : il y avait bien une finalité avérée et l'intensité était réelle puisqu'il fallait mobiliser une charge de deux fois 12,5 kg par une flexion de jambe. Cependant, contrairement à Decety, Jeannerod, Germain et Pastène (1991) et Demougeot et al. (2009), Mulder et al. (2005) n'ont pas observé de modification de l'activité cardiaque, mais seulement une augmentation de la fréquence respiratoire. Alors que l'activité cardiaque variait en fonction de l'intensité de l'effort du mouvement imaginé (locomotion sur tapis roulant) (Decety et al., 1991), elle s'est révélée comparable lors de mouvements du tronc et des jambes (Demougeot et al., 2009). Cette variabilité montre qu'il existe d'autres facteurs d'influence qui ont interféré avec l'imagerie motrice. Un contrôle plus précis des variables indépendantes, incluant les caractéristiques de la tâche et des participants (leur capacité d'imagerie, par exemple) devrait permettre d'isoler les facteurs responsables de la variation cardiaque. Dans l'interprétation de leurs résultats, Mulder et al. (2005) font l'hypothèse que la fréquence cardiaque ne serait pas l'indice le plus sensible à la représentation mentale. Bien que Decety et al. (1991) aient montré que l'imagerie motrice provoquait une activation cardiaque moindre que l'exécution réelle (une locomotion à $12 \mathrm{~km} / \mathrm{h}$ induisait une fréquence cardiaque équivalente à celle d'un déplacement réel à $5 \mathrm{~km} / \mathrm{h}$ ), Mulder et al. (2005) ont fait l'hypothèse que la variabilité de la fréquence pourrait être un indice plus robuste.

Il reste habituel d'enregistrer l'activité cardiorespiratoire comme corrélat de l'imagerie motrice, sans doute à cause de la facilité d'acquisition des données (Collet \& Guillot, 2009, 2010). Le myocarde est doublement innervé par les branches ortho- et parasympathiques. L'imagerie motrice devrait modifier l'activité cardiaque de deux manières : une tachycardie tonique devrait attester l'augmentation de l'éveil physiologique; une bradycardie phasique devrait refléter une focalisation ponctuelle de l'attention. L'imagerie motrice devrait co-activer les deux branches du SNV et, bien que leur réponse soit bien différenciée (tonique vs. phasique), une mesure de fréquence moyenne pourrait atténuer, voire même annuler la réponse résultante. Cet argument méthodologique pourrait justifier la proposition de mesurer la variabilité cardiaque (Mulder et al., 2005) ou traiter le signal cardiaque en extrayant la fréquence instantanée par calcul des intervalles R-R (Collet, Guillot, Lebon, MacIntyre, \& Moran, 2011).

Le choix des indicateurs végétatifs comme variables dépendantes est donc déterminant, de même que celui des tâches motrices support. Elles doivent constituer une «alerte » suffisante pour le système nerveux central afin que sa réponse ait un écho végétatif périphérique. 
L'expérimentateur doit aussi veiller à prévenir le processus d'habituation qui affaiblit puis entraîne l'extinction de la réponse végétative, lorsque la stimulation est répétée.

Même si les études d'Oishi, Kasai et Maeshima (2000) et Oishi et Maeshima (2004) révèlent une élévation des fréquences cardiaques et respiratoires durant l'imagerie, elles s'appuient aussi sur l'enregistrement de l'activité électrodermale (AED). Grâce à deux micro-capteurs placés sur la face palmaire de la main non-dominante, on enregistre l'activité des glandes sudorales eccrines, contrôlées par les terminaisons orthosympathiques. Il n'y a pas d'innervation parasympathique, certainement parce que l'activité sudorale cesse dès lors que la commande sympathique s'arrête. L'intérêt de l'AED est d'avoir un indice sous contrôle orthosympathique unique (exception au principe général de la double innervation). Exprimée en conductance, l'AED augmente pendant la préparation du mouvement et varie parallèlement à l'élévation de la fréquence cardiaque et de la pression artérielle. À la suite de Vissing (1997) qui avait montré que l'activation sympathique de la peau était influencée par les commandes motrices centrales, Critchley (2002) en a déduit que cela rendait l'AED dépendante de la nature de l'information traitée (signification, valence). Ces données vont dans le sens de notre hypothèse de coprogrammation somatique et végétative du mouvement. Ainsi, l'AED constitue un indice psychophysiologique sensible à l'activité cognitive et, en particulier, à l'imagerie motrice. Associée à l'activité cardiaque, l'AED a permis à Guillot et al. (2008) de présélectionner les participants pour une expérience dont l'objectif était d'étudier si les capacités d'imagerie étaient différenciées par des réseaux cérébraux distincts. Lebon, Byblow, Collet, Guillot et Stinear (2012) ont fait de même dans une expérience utilisant la stimulation magnétique transcrânienne. Une évaluation objective des capacités d'imagerie motrice peut s'appuyer sur des mesures de l'activité végétative, tout en étant complétée par d'autres mesures, comme la chronométrie mentale ou les questionnaires d'imagerie (Collet et al., 2011).

Les réponses végétatives induites par la représentation mentale constituent un ensemble de signes objectifs, corrélats de l'activité mentale. Un ensemble de données structurales permet de l'attester. Le contrôle cortical des fonctions végétatives module le rôle automatique et systématique du diencéphale (hypothalamus, en particulier) et du tronc cérébral. Le cortex cingulaire antérieur module l'activité cardiaque via le système sympathique tandis que le contrôle par le cortex médio-ventral règle l'activité vagale (Wong, Massé, Kimmerly, Menon, \& Shoemaker, 2007). Ces deux structures corticales sont aussi connues pour leur médiation des états émotionnels et de la cognition. Elles supervisent l'activité des réseaux de neurones de l'activité électrodermale, dont l'ajustement dépend aussi du cortex préfrontal, insulaire, cingulaire, pariétal, temporal médial, en relation avec les structures limbiques comme l'amygdale et l'hippocampe (Critchley, 2005). Fonctionnellement, ces réseaux déclenchent des réponses végétatives périphériques en réponse à des états affectifs et motivationnels qui vont, à leur tour, conditionner l'activité mentale qu'est l'imagerie motrice. Dans « $\grave{A}$ la recherche du temps perdu», l'évocation de la madeleine par Marcel Proust (1913) lui rappelle les séjours chez sa tante. De ce point de vue, l'affectif est indissociable du cognitif, de l'expérience personnelle rappelée par la mémoire épisodique. La simple évocation mentale des madeleines préparées par sa tante devait sans doute générer, chez M. Proust, de larges réponses végétatives.

L'activité électrodermale, associée à d'autres variables, comme l'activité cardiaque offre donc une méthode inférentielle d'exploration des fonctions mentales supérieures. Elle contribue à l'étude de l'imagerie motrice, comme d'autres méthodes neurophysiologiques et psychologiques (Guillot \& Collet, 2005b).

\section{Conclusion}

L'imagerie mentale est l'une des opérations cérébrales les plus sophistiquées permettant aux personnes d'interroger leurs mémoires, que ce soit celle de la connaissance, de l'expérience ou des savoir-faire et de les alimenter en retour. Lorsqu'elle est motrice, l'imagerie permet d'élaborer des plans d'action, d'anticiper leurs conséquences possibles et, éventuellement, de prévoir leur actualisation. Dans le domaine des relations sociales, nous avons aussi une tendance à simuler ce que les autres pensent de nous à travers la projection mentale, une forme particulière d'imagerie. Ces opérations cérébrales activent des réseaux neuronaux, en partie similaires, et entraînent des modifications physiologiques qui peuvent être enregistrées au niveau des effecteurs périphériques. Elles peuvent être utilisées par les chercheurs pour évaluer indirectement nos états mentaux. S'ils permettent d'évaluer partiellement la qualité du travail mental, l'EMG, les mouvements oculaires et les variables végétatives aident à mieux comprendre les processus sous-jacents des représentations mentales.

Jusqu'à ces deux dernières décennies, le SNV a été plutôt considéré comme un système de contrôle général de l'éveil et de l'activation. Si cette fonction de base reste déterminante à l'efficacité des comportements, les connaissances modernes l'ont élargie jusqu'à en faire un instrument de régulation sociale plus complexe : «L'évolution du système nerveux végétatif fournit un principe organisateur pour interpréter la signification adaptative des mammifères sur le plan affectif, y compris les prises de contact entre congénères et l'établissement de rapports sociaux durables $»$ (Porges, 1998). Chaque opération mentale, dont l'imagerie motrice, se traduit par une activité végétative spécifique, inaccessible à notre volonté et à notre conscience, mais qui la révèle par des variations physiologiques structurées. Leur étude nous ouvre une fenêtre sur une meilleure compréhension du fonctionnement cérébral. 


\section{Bibliographie}

Backs, R.W. (1998). A comparison of factor analytic methods of obtaining cardiovascular autonomic components for the assessment of mental workload. Ergonomics, 41, 733-745.

Bakker, F.C., Boschker, M.S., \& Chung, T. (1996). Changes in muscular activity while imaging weight-lifting using stimulus or response propositions. Journal of Sport and Exercise Psychology, 18, 313-24.

Berntson, G.G., Cacioppo, J.T., \& Quigley, K.S. (1991). Autonomic determinism: the modes of autonomic control, the doctrine of autonomic space and the laws of autonomic constraint. Psychological Review, 98, 459-487.

Beyer, L., Weiss, T., Hansen, E., Wolf, A., \& Seidel, A. (1990). Dynamics of central nervous activation during motor imagination. International Journal of Psychophysiology, 9, 75-80.

Bichat, M.F.X. (1802). General anatomy applied to physiology and medicine. Paris: Brosson, Gabon \& Cie.

Binkofski, F., Amunts, K., Stephan, K.M., Posse, S., Schormann, T., Freund, H.J., Zilles, K., \& Seitz, R.J. (2000). Broca's region subserves imagery of motion: a combined cytoarchitectonic and fMRI study. Human Brain Mapping, 11, 273-285.

Bolliet, O., Collet, C., \& Dittmar, A. (2005a). Observation of action and autonomic nervous system responses. Perceptual and Motor Skills, 101, 195-202.

Bolliet, O., Collet, C., \& Dittmar, A. (2005b). Actual versus simulated preparation in weightlifting: a neurovegetative study. Applied Psychophysiology and Biofeedback, 30, 1120 .

Boschker, M.S.J. (2001). Action-based imagery: on the nature of mentally imagined motor actions. Amsterdam : Ipskamp Printpartners.

Boschker, M.S.J., Bakker, F.C., \& Rietberg, M.B. (2000). Retroactive interference effects of mentally imagined movement speed. Journal of Sports Sciences, 18, 593-603.

Bradley, M.M. (2009). Natural selective attention: orienting and emotion. Psychophysiology, 46, 1-11.

Collet, C., \& Guillot, A. (2009). Peripheral responses elicited by motor imagery: a window on central and peripheral nervous system relationships related to motor inhibition. In S.P. Weingarten \& H.O. Penat (Eds.), Cognitive Psychology Research Developments, 245-259, Nova Science Publishers, 306 pages.

Collet, C., \& Guillot, A. (2010). Autonomic nervous system activities during imagined movements. In A. Guillot \& C. Collet (Eds.), The neurophysiological foundations of mental and motor imagery, Oxford University Press, 95-108

Collet, C., Guillot, A., Bolliet, O., \& Dittmar, A. (2006). Neurovegetative correlates of preparation in weightlifting. International Journal of Sports Physiology and Performance, 1, 373-385.

Collet, C., Guillot, A., Lebon, F., MacIntyre, T., \& Moran, A. (2011). Measuring motor imagery using psychometric, behavioral, and psychophysiological tools. Exercise and Sport Science Review, 39, 85-92.
Conklin, C.A., Tiffany, S.T., \& Vrana, S.R. (2000). The impact of imagining completed versus interrupted smoking on cigarette craving. Experimental and Clinical Psychopharmacology, 8, 68-74.

Critchley, H.D. (2002). Electrodermal responses: what happens in the brain? Neuroscientist, 8, 132-142.

Critchley, H.D. (2005). Neural mechanisms of autonomic, affective, and cognitive integration. Journal of Comparative Neurology, 493, 154-66.

Debarnot, U., Valenza, G., Champely, S., Scilingo, E.G., De Rossi, D., \& Guillot A. (2011). Motor imagery effectiveness for mirror reversed movements. Cognitive, Affective, and Behavioral Neuroscience, 11, 22-31.

Decety, J., Jeannerod, M., Germain, M., \& Pastène, J. (1991). Vegetative response during imagined movement in proportional to mental effort. Behavioural Brain Research, 42, $1-5$.

Decety, J., Jeannerod, M., Durozard, D., \& Baverel, G. (1993). Central activation of autonomic effectors during mental simulation of motor actions in man. Journal of Physiology, 461, 549-63.

Decety, J., Perani, D., Jeannerod, M., Bettinardi, V., Tadary, B., Woods, R.P., Mazziotta, J.C., \& Fazio, F. (1994). Mapping motor representations with positron emission tomography. Nature, 371, 600-602.

Demougeot, L., Normand, H., Denise, P., \& Papaxanthis, C. (2009). Discrete and effortful imagined movements do not specifically activate the autonomic nervous system. PLoS One, 4, e6769.

Deschaumes-Molinaro, C., Dittmar, A., \& Vernet-Maury, E. (1991). Relationship between mental imagery and sporting performance. Behavioural Brain Research, 45, 29-36.

Deschaumes-Molinaro, C., Dittmar, A., \& Vernet-Maury, E. (1992). Autonomic nervous system response patterns correlate with mental imagery. Physiology and Behaviour, 51, 1021-1027.

Dickstein, R., Gazit-Grunwald, M., Plax, M., Dunsky, A., \& Marcovitz, E. (2005). EMG activity in selected target muscles during imagery rising on tiptoes in healthy adults and poststroke hemiparetic patients, Journal of Motor Behavior, 37, 475-83.

Doyon, J., \& Benali, H. (2005). Reorganization and plasticity in the adult brain during learning of motor skills. Current Opinion in Neurobiology, 25, 161-167.

Ehrsson, H.H., Geyer, S., \& Naito, E. (2003). Imagery of voluntary movement of fingers, toes and tongue activates corresponding body-part-specific motor representations. Journal of Neurophysiology, 90, 3304-3316.

Gandevia, S.C., Wilson, L.R., Inglis, J.T., \& Burke, D. (1997). Mental rehearsal of motor tasks recruits alphamotoneurones but fails to recruit human fusimotor neurones selectively. Journal of Neurophysiology, 505, 259-66.

Gao, Q., Duan, X., \& Chen, H. (2011). Evaluation of effective connectivity of motor areas during motor imagery and execution using conditional Granger causality. NeuroImage, $54,1280-1288$. 
Gerardin, E., Sirigu, A., Lehericy, S., Poline, J.B., Gaymard, B., Marsault, C., Agid, Y., \& Le Bihan, D. (2000). Partially overlapping neural networks for real and imagined hand movements. Cerebral Cortex, 10, 1093-1104.

Grèzes, J., \& Decety, J. (2001). Functional anatomy of execution, mental simulation, observation, and verb generation of actions: a meta-analysis. Human Brain Mapping, 12, $1-19$.

Gueugneau, N., Crognier, L., \& Papaxanthis, C. (2008). The influence of eye movements on the temporal features of executed and imagined arm movements. Brain Research, 1187, 95-102.

Guillot, A., \& Collet, C. (2005a). Duration of mentally simulated movement: a review. Journal of Motor Behavior, 37, 10-20.

Guillot, A., \& Collet, C. (2005b). Contribution from neurophysiological and psychological methods to the study of motor imagery. Brain Research Reviews, 50, 387-397.

Guillot, A., \& Collet, C. (2008). Construction of the motor imagery integrative model in sport: a review and theoretical investigation of motor imagery use. International Review of Sport and Exercice Psychology, 1, 31-44.

Guillot, A., Lebon, F., Rouffet, D., Champely, S., Doyon, J., \& Collet, C. (2007). Muscular responses during motor imagery as a function of muscle contraction types. International Journal of Psychophysiology, 66, 1827.

Guillot, A., Collet, C., Nguyen, V.A., Malouin, F., Richards, C., \& Doyon, J. (2008). Functional neuroanatomical networks associated with expertise in motor imagery ability. NeuroImage, 41, 1471-1483.

Guillot, A., Collet, C., Nguyen, V.A., Malouin, F., Richards, C., \& Doyon, J. (2009). Brain activity during visual versus kinesthetic imagery: an fMRI study. Human Brain Mapping, 30, 2157-2172.

Guillot, A., Lebon, F., \& Collet, C. (2010). Electromyographic activity during motor imagery. In A. Guillot \& C. Collet (Eds.), The neurophysiological foundations of mental and motor imagery, Oxford University Press, 83-94.

Guillot, A., Di Rienzo, F. \& Collet, C. (2012). The neurofunctional architecture of motor imagery. In T.D. Papageorgiou, G. Christopoulos \& S. Smirnakis (Eds.), Functional magnetic resonance imaging / Book 1. In Tech.

Guillot, A., Di Rienzo, F., Moran, A., MacIntyre, T., \& Collet, C. (2012). Imagining is not doing but involves motor commands: A review of experimental data related to motor inhibition. Frontiers in Human Neuroscience, 6, 247, 122 .

Guillot, A., Hoyek, N., Louis, M., Collet, C. (2012). Understanding the timing of motor imagery: recent findings and future directions. International Review of Sport and Exercise Psychology, 5, 3-22

Hanakawa, T., Immisch, I., Toma, K., Dimyan, M.A., Van Gelderen, P., \& Hallett, M. (2003). Functional properties of brain areas associated with motor execution and imagery. Journal of Neurophysiology, 89, 989-1002.
Hanakawa, T., Dimyan, M.A., \& Hallett, M. (2008). Motor planning, imagery, and execution in the distributed motor network: a time-course study with functional MRI. Cerebral Cortex, 18, 2775-2788.

Harris, D.V., \& Robinson, W.J. (1986). The effect of skill level on EMG activity during internal and external imagery. Journal of Sport Psychology, 8, 105-11.

Heremans, E., Helsen, W.H., \& Feys, P. (2008). The eye as a mirror of our thoughts: quantification of motor imagery of goal-directed movements through eye-movement registration. Behavioural brain Research, 187, 351-60.

Heremans, E., Nieuwboer, A., Spildooren, J., De Bondt, S., D'hooge, A.M., Helsen, W., \& Feys, P. (2012). Cued motor imagery in patients with multiple sclerosis. Neuroscience, 206, 115-121.

Heremans, E., Smits-Engelsman, B., Caeyenberghs, K., Vercruysse, S., Nieuwboer, A., Feys, P., \& Helsen, W.F. (2011). Keeping an eye on imagery: the role of eye movements during motor imagery training. Neuroscience, 195, $37-44$.

Hesslow, G. (2002). Conscious thought as simulation of behaviour and perception. Trends in Cognitive Science, 6, 242-247.

Holmes, P.S., \& Collins, D.J. (2001). The PETTLEP approach to motor imagery: A functional equivalence model for sport psychologists. Journal of Applied Sport Psychology, $13,60-83$.

Hugdahl, K. (1996). Cognitive influences on human autonomic nervous system function. Current Opinion in Neurobiology, 6, 252-258.

Iacoboni, M., Woods, R.P., Brass, M., Bekkering, H., Mazziotta, J.C., \& Rizzolatti, G. (1999). Cortical mechanisms of human imitation. Science, 286, 2526-2528.

Jackson, P.L., Meltzoff, A.L., \& Decety, J. (2006). Neural circuits involved in imitation and perspective-taking. NeuroImage, 31, 429-439.

Jackson, P.L., Lafleur, M.F., Malouin, F., Richards, C.L., \& Doyon, J. (2003). Functional cerebral reorganization following motor sequence learning through mental practice with motor imagery. NeuroImage, 20, 1171-1180.

Jacobson, E. (1931). Electrical measurement of neuromuscular states during mental activities. American Journal of Physiology, 96, 115-21.

Jeannerod, M. (1994). The representing brain: neural correlates of motor intention and imagery. Behavioral and Brain Sciences, 17, 187-202.

Jennings, J.R., \& van der Molen, M.W. (2005). Preparation for speeded action as a psychophysiological concept. Psychological Bulletin, 131, 434-459.

Jowdy, D.P., \& Harris, D.V. (1990). Muscular responses during mental imagery as a function of motor skill level. Journal of Sport and Exercise Psychology, 12, 191-201.

Kleber, B., Birbaumer, N., Veit, R., Trevorrow, T., \& Lotze, M. (2007). Overt and imagined singing of an Italian aria. Neuroimage, 36, 1238-46. 
Kuhtz-Buschbeck, J.P., Mahnkopf, C., Holzknecht, C., Siebner, H., Ulmer, S., \& Jansen, O. (2003). Effectorindependent representations of simple and complex imagined finger movements: a combined fMRI and TMS study. European Journal of Neuroscience, 18, 3375-3387.

Lafleur, M.F., Jackson, P.L., Malouin, F., Richards, C.L., Evans, A.C., \& Doyon, J. (2002). Motor learning produces parallel dynamic functional changes during the execution and imagination of sequential foot movements. NeuroImage, 2, 142-57.

Laeng, B., \& Teodorescu, D.S. (2002). Eye scanpaths during visual imagery reenact those of perception of the same visual scene. Cognitive Science, 26, 207-231.

Lebon, F., Rouffet, D., Collet, C., \& Guillot, A. (2008). Modulation of EMG power spectrum frequency during motor imagery. Neuroscience Letters, 435, 181-185.

Lebon, F., Byblow, W.D., Collet, C., Guillot, A., \& Stinear, C.M. (2012). The modulation of motor cortex excitability during motor imagery depends on imagery quality. European Journal of Neuroscience, 35, 323-331.

Leonardo, M., Fieldman, J., Sadato, N., Campbell, G., Ibanez, V., Cohen, L., Deiber, M.P., Jezzard, P., Pons, T., Turner, R., Le Bihan, D., \& Hallett, M. (1995). A functional magnetic resonance imaging study of cortical regions associated with motor task execution and motor ideation in humans. Human Brain Mapping, 3, 83-92.

Li, S., Kamper, D.G., Stevens, J.A., \& Rymer, W.Z. (2004). The effect of motor imagery on spinal segmental excitability. Journal of Neuroscience, 27, 9674-80.

Lorey, B., Bischoff, M., Pilgramm, S., Stark, R., Munzert, J., \& Zentgraf, K. (2009). The embodied nature of motor imagery: the influence of posture and perspective. Experimental Brain Research, 194, 233-243.

Lotze, M., \& Halsband, U. (2006). Motor imagery. Journal of Physiology (Paris), 99, 386-395.

Lotze, L., Montoya, P., Erb, M., Hulsmann, E., Flor, H., Klause, U., Birbaumer, N., \& Grodd, W. (1999). Activation of cortical and cerebellar motor areas during executed and imagined hand movements: an fMRI study. Journal of Cognitive Neuroscience, 11, 491-501.

Lotze, M., Scheler, G., Tan, H.R.M., Braun, C., \& Birbaumer, N. (2003). The musician's brain: functional imaging of amateurs and professionals during performance and imagery. NeuroImage, 20, 1817-1829.

Lotze, M., \& Zentgraf, K. (2010). Contribution of the primary motor cortex to motor imagery. In Guillot A. \& Collet C. (Eds.), The neurophysiological foundations of mental and motor imagery, Oxford University Press, 31-46.

Louis, M., Guillot, A., Maton, S., Doyon, J., \& Collet, C. (2008). Effect of imagined movement speed on subsequent motor performance. Journal of Motor Behavior, 40, 117132.

Macuga, K.L., \& Frey, S.H. (2011). Neural representations involved in observed, imagined, and imitated actions are dissociable and hierarchically organized. NeuroImage, 59, 2798-2807.
Malouin, F., Belleville, S., Richards, C. L., Desrosiers, J., \& Doyon, J. (2004). Working memory and mental practice outcomes after stroke. Archives of Physical Medicine and Rehabilitation, 85, 177-183.

Malouin, F., Richards, C., Durand, A., \& Doyon, J. (2008). Reliability of mental chronometry for assessing motor imagery ability after stroke. Archives of Physical Medicine and Rehabilitation, 89, 311-319.

Martin, K.A., Moritz, S.E., \& Hall, C.R. (1999). Imagery use in sport: a literature review and applied model. The Sport Psychologist, 13, 245-268.

Mast, F.W. and Kosslyn, S.M. (2002). Eye movements during visual mental imagery. Trends in Cognitive Sciences, 6, $271-272$.

McCorry, L.K. (2007). Physiology of the autonomic nervous system. American Journal of Pharmaceutical Education, 71, 1-11.

Meister, I.G., Krings, T., Foltys, H., Müller, M., Töpper, R., \& Thron, A. (2004). Playing piano in the mind - An fMRI study on music imagery and performance in pianists. Cognitive Brain Research, 19, 219-228.

Milton, J., Solodkin, A., Hlustîk, P., \& Small, S.L. (2007).The mind of expert motor performance is cool and focused. NeuroImage, 35, 804-813.

Milton, J., Small, S.L., \& Solodkin, A. (2008). Imaging motor imagery: methodological issues related to expertise. Methods, 45, 336-341.

Mogenson, G.J., Jones, D.L., Yim, C.Y. (1980). From motivation to action : functional interface between the limbic system and the motor system. Progress in Neurobiology, 14, 69-97.

Moran, A. (1993). Conceptual and methodological issues in the measurement of mental imagery skills in athletes. Journal of Sport Behavior, 16, 156-70.

Morris, T., Spittle, M., \& Watt, A.P. (2005). Imagery in sport. Champaign, IL : Human Kinetics.

Morrison, S.F. (2001). Differential control of sympathetic outflow. American Journal of Physiology: Regulatory, Integrative and Comparative Physiology, 281, R683-698.

Mulder, T., de Vries S., \& Zijlstra, S. (2005). Observation, imagination and execution of an effortful movement : more evidence for a central explanation of motor imagery. Experimental Brain Research, 163, 344-51.

Munroe, K.J., Giacobbi, P.R., Hall, C., \& Weinberg, R. (2000). The four Ws of imagery use : where, when, why and what. The Sport Psychologist, 14, 119-137.

Munzert, J., Lorey, B., \& Zentraf, K. (2009). Cognitive motor processes : The role of motor imagery in the study of motor representations. Brain Research Reviews, 60, 306-326.

Murphy, S., Nordin, S.M., \& Cumming, J. (2008). Imagery in sport, exercise and dance. In T. Horn (Ed.), Advances in sport psychology Champagne, IL : Human Kinetics, 306315

Oishi K., \& Maeshima T. (2004). Autonomic nervous system activities during motor imagery in elite athletes. Journal of Clinical Neurophysiology, 21, 170-179. 
Oishi, K., Kasai, T., \& Maeshima, T. (2000). Autonomic response specificity during motor imagery. Journal of Physiological Anthropology and Applied Human Science, 19, 255-261.

Paccalin, C., \& Jeannerod, M. (2000). Changes in breathing during observation of effortful actions. Brain Research, 862, 194-200.

Paillard, J. (1982). Apraxia and the neuro-physiology of motor control. Philosophical Transactions of the Royal Society of London, (biology), B298, 111-134.

Personnier, P., Paizis, C., Ballay, Y., \& Papaxanthis, C. (2007). Mentally represented motor actions in normal aging II. The influence of the gravito-inertial context on the duration of overt and covert arm movements. Behavioural Brain Research, 186, 273-283.

Porges, S.W. (1995a). Orienting in a defensive world : mammalian modifications of our evolutionary heritage. A Polyvagal Theory. Psychophysiology, 32, 301-318.

Porges, S.W. (1995b). Cardiac vagal tone: a physiological index of stress. Neuroscience and Biobehavioral Reviews, 19, $225-233$.

Porges, S.W. (1998). Love: an emergent property of the mammalian autonomic nervous system. Psychoneuroendocrinology, 23, 837-861.

Porro, C.A., Cettolo, V., Francescato, M.P., \& Baraldi, P. (2000). Ipsilateral involvement of primary motor cortex during motor imagery. European Journal of Neuroscience, 12, 3059-3063.

Proust, M. (1913). À la recherche du temps perdu. Du côté de chez Swann. Paris : Grasset.

Ranganathan, V.K., Siemionow, V., Liu, J.Z., Sahgal, V., \& Yue, G.H. (2004). From mental power to muscle powergaining strength by using the mind. Neuropsychologia, 42, 944-956.

Rodionov, V., Zislin, J., \& Elidan, J. (2004). Imagination of body rotation can induce eye movements. Acta Otolaryngology, 124, 684-689.

Roland, P.E., Larsen, B., Lassen, N.A., \& Skinhoj, E. (1980). Supplementary motor area and other cortical areas in organisation of voluntary movements in man. Journal of Neurophysiology, 43, 118-136.

Ross, J.S., Tkach, J., Ruggieri, P.M., Lieber, M., \& Lapresto, E. (2003). The mind's eye: Functional MR imaging of golf motor imagery. American Journal of Neuroradiology, 24, 1036-1044.

Ruby, P., \& Decety, J. (2001). Effect of subjective perspective taking during simulation of action: a PET investigation of agency. Nature Neuroscience, 4, 546-50.
Sabate, M., Gonzalez, B., \& Rodriguez, M. (2007). Adapting movement planning to motor impairments: The motorscanning system. Neuropsychologia, 45, 378-386.

Schmidt, R.A. (1975). A schema theory of discrete motor skills learning. Psychological Review, 82, 225-260.

Schwoebel, J., Boronat, C.B., \& Coslett, H.B. (2002). The man who executed "imagined" movements: evidence for dissociable components of the body schema. Brain and Cognition, 50, 1-16.

Sharma, M., Pomeroy, V.M., \& Baron, J.C. (2006). Motor imagery: a backdoor to the motor system after stroke? Stroke, 37, 1941-1952.

Sharma, M., Jones, P.S., Carpenter, T.A., \& Baron, J.C. (2008). Mapping the involvement of BA 4a and 4p during motor imagery. NeuroImage, 41, 92-99.

Slade, J.M., Landers, D.M., \& Martin, P.E. (2002). Muscular activity during real and imagined movement: a test of inflow explanations. Journal of Sport and Exercise Psychology, 24, 151-167.

Sirigu, A., Duhamel, J.R., Cohen, L., Pillon, B., Dubois, B., \& Agid, Y. (1996). The mental representation of hand movements after parietal cortex damage. Science, 273, 15641568 .

Solodkin, A., Hlustik, P., Chen, E.E., \& Small, S.L. (2004). Fine modulation in network activation during motor execution and motor imagery. Cerebral Cortex, 14, 1246-1255.

Tremayne, P., \& Barry, R.J. (2001). Elite pistol shooters: physiological patterning of best vs. worst shots. International Journal of Psychophysiology, 41, 19-29.

Vallbo, A.B., Hagbarth, K.E., \& Wallin, B.G. (2004). Microneurography : how the technique developed and its role in the investigation of the sympathetic nervous system. Journal of Applied Physiology, 96, 1262-1269.

Vissing, S.F. (1997). Differential activation of sympathetic discharge to skin and skeletal muscle in humans. Acta Physiologica Scandinavica, 639, 1-32.

Wallin, B.G., \& Fagius J. (1986). The sympathetic nervous system in man : aspects derived from microelectrodes recordings. Trends in Neurosciences, 2, 63-67.

Wuyam, B., Moosavi, S.H., Decety, J., Adams, L., Lansing, R.W., \& Guz A. (1995). Imagination of dynamic exercise produced ventilatory responses which were more apparent in competitive sportsmen. Journal of Physiology (London), 482, 713-724.

Wong, S.W., Massé, N., Kimmerly, D.S., Menon, R.S., \& Shoemaker, J.K. (2007). Ventral medial prefrontal cortex and cardiovagal control in conscious humans. NeuroImage, 35, 698-708. 